\title{
Human reliability research of numerical control machining based on optimized Holistic Decision Tree
}

\author{
Lu Chen ${ }^{1,2}$, Run-Jiang Zhao ${ }^{1}$, Jian Jiao ${ }^{1, a}$ and Yan-Bo Zhang ${ }^{1}$ \\ ${ }^{1}$ School of Reliability and Systems Engineering, Beihang University, China \\ ${ }^{2}$ China Astronautics Standards Institute, China
}

\begin{abstract}
Human reliability has been a focus in system reliability and safety researches since 1960s, and now human factor has become an increasingly important reason which affects system reliability. Numerical control machining system characterized by man-machine interaction can be applied to automatic parts machining with high efficiency. However, professional and technical staffs are still needed for clamping, positioning, inspection and others. So there is no doubt that the quality of the parts might be influenced by personnel behaviour. What's more, on-site staff is responsible for handling potential fault directly. The study on human error plays an important role to ensure the quality and reduce industrial accident. Holistic Decision Tree (HDT) is a dynamic Human Reliability Analysis (HRA) method, emphasizing the wholeness of man-machine interaction. This paper tries to use an optimized HDT method to investigate human reliability in numerical control machining system. In order to obtain a rational order of the importance of each IF, this paper incorporates entropy weight method to the conventional expert method to determine the relative weight value of each human behaviour influence factor (IF), and finally calculates the human error probability (HEP).
\end{abstract}

\section{Introduction}

Numerical control machining system is a typical complicated man-machine interaction system, which needs relevant professional and technical staff for clamping, positioning, inspection and others, so there is no doubt that the quality of the parts produced by the system might be influenced by personnel behaviour. What's more, on-site staff is responsible for handling potential faults directly, and human error also plays an important role to ensure the parts quality and reduce industrial accident. However, the existing studies on the reliability of the numerical control machining system mostly focus on equipment failures and the improvement of the man-machine interface [1-3].

Human reliability, a term used to express personnel performance, can be described as the probability of personnel completing a task successfully within the specified minimum time limit (if there have time requirements), and at any operation stage of the system [4]. The research on human reliability analysis (HRA) began in the 1950s [5]. In the late 1950s and early 1960s, researchers started to discuss the influences of human error on the system reliability, and there are more than twenty types of HRA methods up to now. The first generation HRA methods such as Technique for

\footnotetext{
${ }^{a}$ Corresponding author : jiaojian@buaa.edu.cn
}

(C) The Authors, published by EDP Sciences. This is an open access article distributed under the terms of the Creative Commons Attribution License 4.0 (http://creativecommons.org/licenses/by/4.0/). 
Human Error Rate Prediction (THERP) [6], Human Error Assessment and Reduction Technique (HEART) [7] and Success Likelihood Index Method (SLIM) [8] are widely used in risk analysis of industrial system. However, there are some insufficiencies in the consideration of human cognitive error and situational factors. Nevertheless, the effect of environment or work scenario on human behaviour is generally investigated in the second generation HRA methods such as A Technique for Human Error Analysis (ATHEANA) [9], Cognitive Reliability and Error Analysis Method (CREAM) [10] and Holistic Decision Tree (HDT) [11]. ATHEANA and CREAM focuses mainly on retrospective analysis and predictive analysis. The analytical processes are complicated and a large amount of data resource input is required, which limits their applicability and practicality in industrial production field.

As a dynamic HRA method, Holistic Decision Tree (HDT) method was proposed based on simulator's analysis results of the "Paks" nuclear power station by A.J. Spurgin [12] in the 1990s, emphasizing the wholeness of man-machine interaction. In the form of decision tree, various behaviour influence factors (IFs) are considered to judge their affects the human error probability in this method. In recent years, researchers tried to apply the HDT method to other man-machine interaction industries and fields, such as air traffic control, aircraft maintenance, automatic drive [1315 ] etc. Lots of qualitative and quantitative conclusions got from HDT analysis have been adopted to improve man-machine system design, development and to reduce human errors.

This paper tries to use an optimized HDT method to investigate behaviour Ifs, such as mental states of the operators, personnel skills, man-machine interface, working environment, operation specification, etc., to analyze and assess human reliability in Numerical control machining system. In order to obtain a rational order of the importance of each IF, this paper incorporates the objective entropy weight method to the conventional expert method to determine the relative weight value of each IF, and finally calculates the human error probability (HEP), which can provide reasonable and reliable basis for making preventive and control measures for the potential accident or production damage. This optimized HDT method is more objective and efficient, and is available for improving operational efficiency and operational safety of numerical control machining industry.

\section{Principle of the HDT method}

\subsection{Basic principle of the HDT method}

The basic principle of HDT is that accident scenarios and staffs' situation are used to determine HEP. The HDT model can be expressed as the following equations $[5,16]$, which can link staffs' scene with HEP.

Human error probability = Function of the staffs' situation

Staffs' situation $=$ Function of the influence factors

Human error probability $=$ Function of the influence factors (man-machine interface, leadership quality, etc.)

A typical decision tree is constituted by some main elements such as IFs, branch-like structure, weight value of each IF and quality description value etc., as shown in Figure 1. The titles of the X axis are IFs, along with the corresponding weight value of each scenario. The notes on the tree's branches are quality description (QD) levels, along with the corresponding quality values (QV). QV is the quantified expression of QD levels, which describes the increasing degree of HEP accelerated by the corresponding QD. Just like building an Event Tree, the structure of the HDT is divided into several paths according to the titles of the $\mathrm{X}$ axis. The branch value is corresponding to different IF's QD level, and each path is corresponding to a different combination of quality descriptions.

\subsection{Principle of the entropy weight method}

The concept of entropy was produced in thermodynamics, which is used to describe the phenomenon of irreversible process. In 1948, C.E. Shannon introduced the concept of entropy into information 
theory as a measure parameter. According to the basic principle of information theory, information is used to measure the order degree of a system while entropy measures disorder. The increasing of information means the decreasing of entropy; and information is inversely proportional to entropy [17, $18]$.

As an objective way to determine the weighted value of indicators, entropy weight method can use multiple indicators to evaluate multiple objects, and has the following three characteristics [19]: (1) Entropy weight method, based on the "differentia-driven" theory, highlights the local differences and obtain the optimal weight value through the actual data of all samples, which avoids the influence of human error, and has high confidence level; (2) The weight process has transparency and reproducibility; (3) Normalization method is used to deal with the dimensionless processing of data, which has a good robustness and can keep monotonicity, scaling of independence and total constancy.

\section{The optimized Holistic Decision Tree}

\subsection{Procedure of HDT analysis}

The general procedure of HDT contains 9 steps:

(1) List all of the possible influence factors (IFs).

(2) Distinguish between IFs for overall situation and IFs for related scenarios. The value of human error probability is influenced by the scenario (local) and global factors, where the latter have influence on every scenario.

(3) Arrange the order of the IFs according to their importance, and select the most important one.

(4) Scrupulously select and define the quality description levels of these factors, and draw the tree structure.

(5) Determine the weighted value of IFs. Expert judgment method is recommended to get the weighted value, and different participants' judgment can be used as the measurement of uncertainty.

(6) Select the minimum and maximum calibration values of human error probability.

(7) Rank each IFs of each scenario with quality description (QD).

(8) Determine the corresponding quality values (QV) of QD.

(9) Calculate of human error probability (HEP).

\subsection{Incorporate entropy weight into the expert method}

Entropy weight method $[20,21]$ is an objective method based on entropy calculation of each IF to measure the amount of information, and then to determine the weight value. Weighting each evaluation index with entropy weight can avoid human interference, which makes the evaluation results more objective. Entropy weight method ensures that the established indicators can reflect most of the original information, so that the result is more in line with the actual situation.

Expert method integrates experts' industry experience of all relevant fields to give each IF a weight value. Because it is the subjective judgment of decision makers, the weight value may be more or less affected by the lack of experience or knowledge of decision makers. Therefore, this paper tries to incorporate entropy weight method to the conventional expert method to determine the relative weight value of each IF to improve its accuracy.

Firstly, we can get a $n \times m$ matrix of the original data from the determined $n$ different samples and $m$ evaluation indexes, as follows:

$$
X=\left[\begin{array}{cccc}
x_{11} & x_{12} & \cdots & x_{1 n} \\
x_{21} & x_{22} & \cdots & x_{2 n} \\
\vdots & \vdots & \vdots & \vdots \\
x_{n 1} & x_{n 2} & \cdots & x_{n m}
\end{array}\right]_{n \times m}
$$


where $x_{\mathrm{ij}}$ means the $j_{\mathrm{th}}$ evaluation index of the $i_{\text {th }}$ sample, $i=1,2, \ldots, n ; j=1,2, \ldots, m$.

Due to the different dimensions of the various indexes, normalization is needed for each index. Take the ratio between column vector and the sum of all elements in the matrix $X$ as the normalized value, and its computation formula is as follows:

$$
z_{i j}=\frac{x_{i j}}{\sum_{j=1}^{m} x_{i j}}
$$

where $z_{i j}$ are the elements of the normalized matrix.

The computation formula to determine the entropy value of evaluation index is as follows:

$$
H\left(x_{j}\right)=-k \sum_{i=1}^{n} z_{i j} \ln z_{i j}
$$

where $k$ is the adjustment coefficient; $z_{i j}$ means the $j_{t h}$ index's standardized value of the $i_{t h}$ evaluation unit, $i=1,2, \ldots, n ; j=1,2, \ldots, m$.

Then, transform the entropy value of evaluation index into weighted value $w 1_{j}$.

$$
w 1_{j}=\frac{1-H\left(x_{j}\right)}{m-\sum_{j=1}^{m} H\left(x_{j}\right)}
$$

where $0 \leq w 1_{j} \leq 1, \sum_{j=1}^{m} w 1_{j}=1$.

Finally, incorporate the weighted value $w 1_{j}$ determined by entropy weight method to the weighted value $w 2_{j}$ determined by experts to obtain the final weighted value $w_{j}$.

$$
w_{j}=\alpha \cdot w 1_{j}+(1-\alpha) \cdot w 2_{j}, 0<\alpha<1
$$

\subsection{Calculation of the human error probability (HEP)}

After many times practice in recent years, Spurgin and his partners adopted the following logarithmic interpolation formula to calculate HEP:

$$
\begin{gathered}
S_{i}=\sum_{i=1}^{n}\left(Q V_{i}\right) I_{i} \\
\ln \left(H E P_{i}\right)=\ln \left(H E P_{l}\right)+\ln \left(H E P_{h} / H E P_{l}\right)\left(\frac{S_{i}-S_{l}}{S_{h}-S_{l}}\right)
\end{gathered}
$$

Thereinto,

$S_{i}$ : The $i_{\text {th }}$ path weighted value. $S_{i}$ is the weighted value of each IFs of the corresponding path's human error probability. We considered that factors of the path are work multiplied when calculating the weighted value $S_{i}$. Assuming the weighted value of each IF after normalization as $I_{i}\left(i=1 \sim n, n\right.$ is the number of IFs), $I_{i}$ corresponds to the quality description value $Q V_{i}$;

$S_{l}$ : Minimum path weighted value;

$S_{h}$ : Maximum path weighted value;

$H E P_{i}:$ The $i_{\text {th }}$ path's human error probability;

$H E P_{l}$ : Minimum calibration value of human error probability;

$H E P_{h}$ : Maximum calibration value of human error probability. 


\section{Case study}

\subsection{Introduction of the numerical control machining industry}

Originating from the needs of aviation industry, numerical control technology is a kind of machining method using digital information control parts and cutters' displacement. The control system gives commands and instructions to make cutting tools do all kinds of cutting motion conforming to the requirements. Numerical control machine will express the shape and size of the workpiece and other technical requirements and process requirements in the form of numbers and letters. It's an effective way to address parts machining problems about changing varieties, small batch, complex shape and high precision and to realize automatic processing efficiently.

However, professional and technical staffs are still needed for clamping, positioning, inspection and other duties. So there is no doubt that the quality of the parts might be influenced by personnel behaviour. What's more, on-site staff is responsible for handling potential fault directly. The study on human error plays an important role to ensure the production quality and reduce industrial accident. This paper will take the numerical control operators as case study to investigate human reliability by using the optimized HDT proposed in Section 3.

\subsection{Select the behaviour IFs of staffs}

The main duties of numerical control personnel are listed as follow: checking machines before starting in accordance with the operating rules; clamping, positioning and a series of preparations before processing; determining the processing status; operating numerical control machine tool parts; loading \& un-loading parts and tooling. IFs that affect the operating personnel are selected as follow: mental state of the operators, personnel skills of the operators, quality of the man-machine interface, working environment of the workshop, operation specification of the workshop.

These IFs are analyzed to classify operators' behaviour factors accurately and the QD levels of various Ifs are determined.

(1) Mental state of the operators

Mental state of the operators (non-capacity factors) refers to work attitude and professionalism of the operators, which means whether the operating personnel's subjective initiative is at play. In this case, mental state of the operators includes two levels: good and poor.

(2) Personnel skill of the operators

Personnel skill of the operators (capacity factors) refers to individual psychological quality, physiological quality, professional skill and the operator training. Only the strong personal psychological quality can keep up in an emergency, and make the right decision. Only a good physiological quality and healthy body can competent to shift work for a long time. Training can improve the operation personnel's understanding of numerical control machining and the function of machine tools, and enhance operators' processing experience. Operators training can also improve the understanding of safe operate of machine tools. In this case, personnel skill of the operators is classified into three levels: skilled, enough and lack.

(3) Quality of the man-machine interface

Large numerical control workshop has different requirements on parts, and needs different numerical control machine tools. The numerical control machine tools manufactured by different manufacturers vary widely, so the degree of friendly man-machine interface has a great influence on operator's behaviour. A good man-machine interface can offer distinct content display, sensitive alarm display and fluent information transfer, while a bad interface of some relatively old machine may not supply enough information, and can lead to some wrong operation. In this case, quality of the manmachine interface is divided into three levels: helpful, available and adverse.

(4) Working environment of the workshop

Working environment of the workshop refers to environmental noise, high and low temperature, lighting, space and other effects on operator. Some measures such as noise reduction, the installation 
of air conditioning system, supplying bright and spacious workshop environment can effectively improve operator's work ability. In this case, working environment of the workshop is divided into two levels: comfortable and uncomfortable.

(5) Operate specification of the workshop

The machining of a large numerical control machine is generally a complicated process where a tiny mistake may produce great loss. Thus, operators have to follow a series of operation specification, including boot checking specification, equipment cleaning specification and processing steps inspection, etc. In this case, operate specification of the workshop is classified into two levels: helpful and available.

\subsection{Rank the importance of each IF}

After the IFs are selected, the next step is to determine the relative weighted value of them. Table 1 incorporate entropy weight method to the conventional expert method to determine the relative weight value of each IF, where in accordance with the distribution of 4:6. Relevant data come from a manufacturing plant of aviation products.

Table 1. Weight values of IFs.

\begin{tabular}{|c|c|c|c|c|c|}
\hline IFs & $\begin{array}{c}\text { Mental state of } \\
\text { the operators }\end{array}$ & $\begin{array}{c}\text { Personnel } \\
\text { skill of the } \\
\text { operators }\end{array}$ & $\begin{array}{c}\text { Quality of the } \\
\text { man-machine } \\
\text { interface }\end{array}$ & $\begin{array}{c}\text { Working } \\
\text { environment of } \\
\text { the workshop }\end{array}$ & $\begin{array}{c}\text { Operate } \\
\text { specification of } \\
\text { the workshop }\end{array}$ \\
\hline $\begin{array}{c}\text { Entropy weight } \\
\text { method }\end{array}$ & 0.44 & 0.37 & 0.09 & 0.08 & 0.02 \\
\hline $\begin{array}{c}\text { Expert } \\
\text { Method }\end{array}$ & 0.45 & 0.35 & 0.10 & 0.05 & 0.05 \\
\hline $\begin{array}{c}\text { Comprehensive } \\
\text { weights }\end{array}$ & 0.446 & 0.358 & 0.096 & 0.062 & 0.038 \\
\hline
\end{tabular}

\subsection{Select quality factors \& identify the importance of IFs}

The IFs can be described into three QD levels in practical analysis generally. If it is divided into two levels, the results are slightly rough; if it is divided into four levels, the middle two levels may be difficult to distinguish in actual operation.

In this case, there are five IFs. If the QD of each IF is divided into three levels, the structured HDT will have $3^{5}=243$ branches in theory; if the QD of each IF is divided into two levels, the structured decision tree will have $2^{5}=32$ branches. A huge decision tree will obstruct the analysis while a small one cannot supply enough information, so an appropriate size should be determined carefully. According to the actual situation, different divisions on the QD of the five IFs are chosen as follows:

(1) Mental state of the operators: good and poor.

(2) Personnel skill of the operators: skilled, enough and lack.

(3) Quality of the man-machine interface: helpful, available and adverse.

(4) Working environment of the workshop: comfortable and uncomfortable.

(5) Operate specification of the workshop: helpful and available.

\subsection{Select quality description value}

After the determination of the QD of each IF, HDT method focuses on how the different qualities affect reliability. Define a unified value "1" for the best QD level (such as good, skilled and helpful) of each IF. Lower QD level will increase the human error probability, which can be transformed as quality factor values greater than " 1 ". This paper use the order quantity " $1 \sim 9$ " to compare and measure the importance of quality factor with reference to the analytic hierarchy process (AHP). "3" is selected as intermediate gradient factor to measure the quality description level from good to middle 
and from middle to poor. Therefore, in this case, the QVs of the IFs (shown in Table 2) are expressed as: "1" for good, " 3 " for middle and "9" for poor.

Table 2. Quality description (QD) levels and corresponding quality values (QV) of the IFs.

\begin{tabular}{|l|c|c|c|c|c|}
\hline $\begin{array}{c}\text { QD levels of } \\
\text { IFs }\end{array}$ & $\begin{array}{c}\text { Mental state of } \\
\text { the operators }\end{array}$ & $\begin{array}{c}\text { Personnel skill of } \\
\text { the operators }\end{array}$ & $\begin{array}{c}\text { Quality of } \\
\text { man-machine } \\
\text { interface }\end{array}$ & $\begin{array}{c}\text { Working } \\
\text { environment of } \\
\text { the workshop }\end{array}$ & $\begin{array}{c}\text { Operate } \\
\text { specification of } \\
\text { the workshop }\end{array}$ \\
\hline "1" for good & good & skilled & helpful & comfortable & helpful \\
\hline "3" for middle & poor & enough & available & uncomfortable & available \\
\hline "9" for poor. & - & lack & adverse & - & - \\
\hline
\end{tabular}

\subsection{Construct the Holistic Decision Tree (HDT)}

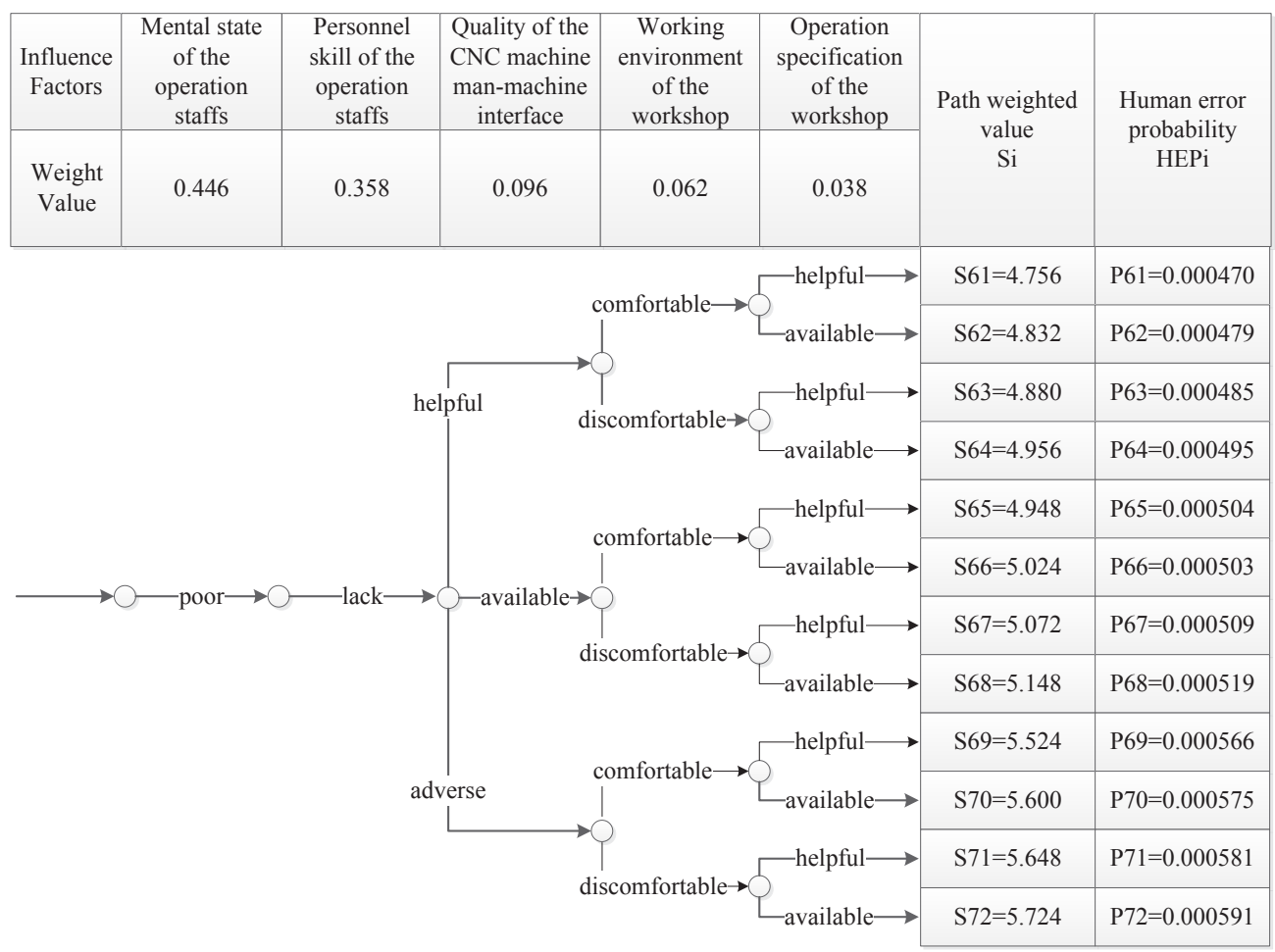

Figure 1. Last part of the HDT.

A typical HDT is constructed using some main elements such as the list of the key IFs, the relative weight of each IF, the list of the QD factors and corresponding QVs, and the calibration values. Assume that IFs are relatively independent, so they can arbitrarily arranged in the decision tree. After the decision tree's structure is built up, human error probability values can be calculated according to the relative weight, quality value, and the calibration value. It can be concluded from the typical decision tree that the lower position in the tree, the higher HEP value is. Thus, the HEP values at upper and lower boundary respectively are corresponding to the best and the worst IF's quality description respectively, and those two values are called the calculated calibration values.

The HDT is constructed according to the division of quality with a total of 72 branches. Figure 1 shows the last part of the HDT with the highest HEP value. 


\subsection{Select the calibration values of human error probability}

Maximum calibration value of HEP assumes that all of the IFs are on the worst level, and staffs often make mistakes. If fail to get any help in the accident, the failure rate is 1 . Minimum calibration value of HEP assumes that all of the IFs are on the best level. The minimum calibration values can change, and its uncertainty can be included in the analysis. In most scenarios, the $5 \%$ quantile of minimum calibration value is set as $10^{-4}$, while $95 \%$ quantile is set as $10^{-3}$; In some slight severe cases, the $5 \%$ quantile of minimum calibration value is set as $10^{-3}$, and $95 \%$ quantile is $10^{-2}$; In some serious cases, the $5 \%$ and $95 \%$ quantile of minimum calibration value are $10^{-2}$ and 0.1 respectively. So, in this case, we set the $5 \%$ quantile of minimum calibration value as $10^{-4}$, and $95 \%$ quantile as $10^{-3}$.

\subsection{Calculate the human error probability (HEP)}

After determining the weight value, the quality description value and the calibration value of the IFs, the HEP of each path can be figured out.

First, the $i_{\text {th }}$ path weighted value $S_{i}$ is calculated through formula (6). $S_{i}$ is the weighted value of each IF for HEP in the corresponding path. Then, the HEP value $H E P_{i}$ can be figured out through formula (7). As shown in the last two columns of Figure 1, the HEP rises along with the worse of quality description level.

\subsection{Discuss the analysis results}

Resourcing historical statistics of human error of numerical control machining workshop's accident investigation reports, the average QVs of each IF were got. In which, mental state of the operators is usually good, recommended value is 1; personnel skill of the operators is usually enough, recommended value is 3; quality of man-machine interface is usually available, recommended value is 3 ; working environment of the workshop is usually comfortable, recommended value is 1; operate specification of the workshop is usually helpful, recommended value is 1. Querying the constructed HDT and combining with the above results we have gotten, the quality description value of the five IFs and the final HEP value under the usual scene's HDT path is figured out as 0.000114 , as shown in Table 3.

Table 3. HEP value under usual scene's path.

\begin{tabular}{|c|c|c|c|c|}
\hline Influence factors (IFs) & $\begin{array}{c}\text { Weighted } \\
\text { value of IFs(Ij) }\end{array}$ & $\begin{array}{c}\text { Quality description } \\
\text { (average level) }\end{array}$ & $\begin{array}{c}\text { Quality } \\
\text { value }(\boldsymbol{Q V} \boldsymbol{i})\end{array}$ & $(\boldsymbol{Q V} \boldsymbol{i}) \boldsymbol{I j}$ \\
\hline Mental state of the operators & 0.446 & Fine (good) & 1 & 0.446 \\
\hline Personnel skill of the operators & 0.358 & Enough (middle) & 3 & 1.074 \\
\hline Quality of man-machine interface & 0.096 & Available (middle) & 3 & 0.288 \\
\hline Working environment of the workshop & 0.062 & Comfortable (good) & 1 & 0.062 \\
\hline Operate specification of the workshop & 0.038 & Helpful (good) & 1 & 0.038 \\
\hline Path weighted value $\left(\boldsymbol{S}_{\boldsymbol{i}}\right)$ & - & - & - & 1.908 \\
\hline Human error probability $(\boldsymbol{H E P})$ & - & - & - & $\mathbf{0 . 0 0 0 1 1 4}$ \\
\hline
\end{tabular}

Through the HDT analysis, it can be concluded that personnel skill and mental state of the operators are the two most critical IFs in numerical control machining industry. Especially, the lack of operative skill can accelerate the increasing degree of HEP by $56.3 \%$ while the poor mental state of the operators accelerates the increasing degree of HEP by $23.4 \%$. Based on the analysis results, it is recommended that more professional training is required to enrich operators' skills and overtime 
working should be decreased to ensure a good mental state of the operators to reduce the human error probability.

\section{Conclusions}

Operators' behaviour influence factors (IFs) and their relationship in the numerical control machining industry is analyzed based on the optimized HDT proposed in this paper. Human error probability (HEP) values under different scenario are figured out and the two most critical IFs in numerical control machining industry is found out, which are personnel skill and mental state of the operators. Suggestion and measure for improvement are given specifically for purpose of the reduction of HEP. This optimized HDT method can provide reasonable and reliable basis based on analysis and calculation results for making the prevention and control measures of product damage and accident, and then improve the efficiency and safety of the industrial production process.

\section{References}

1. X.C. He, International Journal of Advanced Manufacturing Technology, 85(1-4), 115-131 (2016)

2. T.D. Huang, et al., Journal of Shanghai Jiaotong University, 21(5), 635-640 (2016)

3. X. Wu, Research on the Reliability Analysis and Growth of the CNC Machining Center, Journal of Guangxi University for Nationalities (2016)

4. B.S. Dhillon, Human Reliability: With Human Factors (Oxford, UK, 1986)

5. X.H. He and X.R. Huang, Human reliability analysis in engineering system: theory, methods and applications (Beijing, China, 2007)

6. A.D. Swain and H.E. Guttman, Handbook of human reliability analysis with emphasis on nuclear power plant applications, Technical Report (1983)

7. J.C. Williams, Proceedings of the IEEE 4th conference on human factor in power plants, June 6-9, (1985)

8. D.E. Embrey, P.Humphreys and E.A. Rosa, et al., SLIM-MAUD: an approach to assessing human error probabilities using structured expert judgment, Volume I (1984)

9. S.E. Cooper, et al., A technique for human error analysis (ATHEANA), Office of Scientific \& Technical Information Technical Reports, NUREG/CR-6350 (1996)

10. E. Hollnagel, Cognitive Reliability and Error Analysis Method CREAM (1998)

11. A.J. Spurgin, Frontiers Science Series, 2, 1343-1350 (2000)

12. A.J. Spurgin and M.V. Frank, Developments in HRA technology from nuclear to aerospace Probabilistic Safety Assessment and Management (London, UK, 2004)

13. H.F. Li, W.Q. Li and X.P. Wu, Chinese Journal of Ergonomics, 2, 34-39 (2010)

14. X.Q. Zhang and H. Wang, China Safety Science Journal, 22(1), 100-105 (2012)

15. L.Q. Lin, K.M. Huang and S.J. Liao, Communications Standardization, 42(19), 75-80 (2014)

16. F. Sun, S. Zhong and Z.Y. Wu, Chinese Journal of Nuclear Science and Engineering, 28(3), 268$272(2008)$

17. D.L. Mon, C.H. Cheng and J.C. Lin, Fuzzy Sets and Systems, 62, 127-134 (1994)

18. Y. Fu, X.P. Wu and Q. Ye, ACTA Electronica Sinica, 38(7), 1489-1494 (2010)

19. L.J. Sun, X.J. Xing and D.Q. Zhou, Statistics and Decision, 21, 153-154 (2010)

20. W. Li, S.W. Gong and X.B. Li, China Safety Science Journal, 18(3), 166-170 (2008)

21. S. Zhang, M. Zhang and G.T. Chi, Chinese Journal of Management, 1, 34-42 (2010) 